\title{
The mycelium-associated Streptomyces reticuli catalase-peroxidase, its gene and regulation by FurS
}

Universität Osnabrück, FB Biologie/Chemie, Barbarastraße 11, 49069 Osnabrück, Germany

\author{
Pei-jian Zou, llya Borovok, Darío Ortiz de Orué Lucana, Dagmar Müller \\ and Hildgund Schrempf
}

\author{
Author for correspondence: Hildgund Schrempf. Tel: +49541969 2895. Fax : +495419692804. \\ e-mail: schrempf@sfbbio1.biologie.uni-osnabrueck.de
}

\begin{abstract}
During early stages of growth, Streptomyces reticuli synthesizes a hyphaeassociated, haem-containing enzyme which exhibits catalase and peroxidase activities with broad substrate specificity (CpeB). The purified dimeric enzyme $(160 \mathrm{kDa})$ consists of two identical subunits. Using anti-CpeB antibodies and an expression- as well as a mini-library, the corresponding сpeB gene was identified and sequenced. It encodes a protein of $\mathbf{7 4 0}$ aa with a molecular mass of $81.3 \mathrm{kDa}$. The deduced protein shares the highest level of amino acid identity with KatG from Caulobacter crescentus and Mycobacterium tuberculosis, and PerA from Bacillus stearothermophilus. Streptomyces lividans transformants carrying $\triangle \mathrm{Cp} B$ and the upstream-located furS gene with its regulatory region on the bifunctional vector PWHM3 produced low or enhanced levels of CpeB in the presence or absence of Fe ions, respectively. An in-frame deletion of the major part of furS induces increased CpeB synthesis. The data imply that FurS regulates the transcription of $c p e B$. The deduced FurS protein is rich in histidine residues, contains a putative $\mathbf{N}$-terminally situated helix-turn-helix motif and has a molecular mass of $15.1 \mathrm{kDa}$. It shares only $29 \%$ amino acid identity with the Escherichia coli ferric uptake regulator (Fur) protein, but about $64 \%$ with FurA deduced from the genomic sequences of several mycobacteria. The predicted secondary structures of FurS and FurA are highly similar and considerably divergent from those of the $E$. coli Fur. In contrast to some Gram-negative bacteria, within several mycobacteria an intact furA gene or a furA pseudogene is upstream of a catalase-peroxidase (katG) gene predicted to encode a functional or a non-functional (Mycobacterium leprae) enzyme. Thus the data obtained for Streptomyces reticuli are expected to serve as an additional model to elucidate the regulation of mycobacterial catalase-peroxidase genes.
\end{abstract}

Keywords: Fur proteins, catalase-peroxidase genes, streptomycetes, mycobacteria

\section{INTRODUCTION}

The dismutation of $\mathrm{H}_{2} \mathrm{O}_{2}$ to $\mathrm{O}_{2}$ and $\mathrm{H}_{2} \mathrm{O}$ is catalysed by catalases, whereas peroxidases use $\mathrm{H}_{2} \mathrm{O}_{2}$ to oxidize a variety of compounds (Elstner, 1982). Catalases are

Abbreviations: ABTS, 2,2'-azino-bis(3-ethylbenzthiazoline-6-sulfonic acid; 4C1N, 4-chloro-1-naphthol.

The GenBank/EMBLDDBJ accession number for the sequence determined in this paper is $Y 14317$. protohaem IX proteins of $225-270 \mathrm{kDa}$, which consist of four identical subunits. The enzymes are active within a broad $\mathrm{pH}$ range, resistant to treatment with ethanol and specifically inhibited by 3-amino-1,2,4-triazole (Nadler et al., 1986; Margoliash et al., 1960). Bifunctional catalase-peroxidases constitute a distinct group of proteins exhibiting catalase and peroxidase activities. The ratio of these activities varies among the members of this class of enzymes.

Most microbial catalase-peroxidases contain two or four identical subunits and they carry ferric proto- 
porphyrin IX (0.5-1 molecule per subunit) as the prosthetic group. Monomeric catalase-peroxidases have been purified from two halophilic archaea (Cendrin et al., 1994; Fukumori et al., 1985).

Proteins deduced from the few known characterized catalase-peroxidase gene sequences share varying but high levels of amino acid identity, but show little sequence similarity with proteins derived from typical catalase genes. It is interesting that bacterial catalaseperoxidases are homologous to eukaryotic, monomeric, haem-containing peroxidases comprising 290-350 aa. Analyses of deduced proteins indicate that the double length of the bacterial enzymes can be attributed to gene duplication (Welinder, 1991). A study of the crystal structure of cytochrome $c$ peroxidase (Finzel et al., 1984) and modelling data led to the suggestion that the $\mathrm{N}$ terminal part, but not the C-terminal half of a subunit from a bacterial catalase-peroxidase binds haem (Welinder, 1991).

In Escherichia coli, protection against $\mathrm{H}_{2} \mathrm{O}_{2}$ involves the enzymes hydroperoxidase HPII (a typical catalase) and hydroperoxidase HPI (an atypical catalase corresponding to a catalase-peroxidase type) that are encoded by $k a t E$ and $k a t G$, respectively. Transcription of $k a t E$ requires the sigma factor $\sigma^{\mathrm{s}}$ and occurs when cultures enter stationary phase (Loewen \& Hengge-Aronis, 1994). In E. coli and Salmonella typhimurium, in contrast, $k a t G$ is transcribed independently of $\sigma^{\mathrm{s}}$, in response to $\mathrm{H}_{2} \mathrm{O}_{2}$ and under the control of the positive activator OxyR. OxyR senses oxidative stress (Storz et al., 1990) and is regulated by reversible intramolecular disulphide bond formation (Zheng et al., 1998). The regulation of catalase-peroxidase genes from various other micro-organisms has up to now not been elucidated in detail.

In this paper we report on the characteristics of a mycelium-associated Streptomyces reticuli catalase-peroxidase with broad substrate specificity (CpeB) and on the corresponding gene regulated by the newly described gene furS.

\section{METHODS}

Bacterial strains and plasmids. Streptomyces reticuli $\mathrm{Tü45}(\mathrm{H}$. Zähner, Tübingen, Germany) and Streptomyces lividans 66 (D. A. Hopwood, John Innes Institute, Norwich, UK) were used. The plasmids pWHM3 (Vara et al., 1989) and pUC18/ pUC19 (Sambrook et al., 1989) were gifts from C. R. Hutchinson (University of Wisconsin, Madison, USA) and J. Messing (State University of New Jersey, Piscataway, USA), respectively. A derivative of pUC18 (named pUDS18) carrying a deletion of the SstI site was constructed after SstI cleavage, Klenow treatment and religation.

Media and culture conditions. For cultivation of Streptomyces strains, complete and minimal media were used (Schlochtermeier et al., 1992a). Minimal medium was supplemented with glucose or Avicel $(1 \%)$. Depending on the purpose of the experiments, cultures were grown in baffled Erlenmeyer flasks containing $5-200 \mathrm{ml}$ on a rotary shaker for 2-8 d. E. coli strains (JM83 or XL-1 Blue) were cultivated in LB medium at $37^{\circ} \mathrm{C}$ (Sambrook et al., 1989).

Chemicals and enzymes. Chemicals for SDS gel electrophoresis were obtained from Serva. Molecular mass markers, nitrophenyl, O-dianisidine, 4-chloro-1-naphthol (4C1N) and 2,2'-azino-bis(3-ethylbenzthiazoline-6-sulfonic acid) (ABTS) were supplied by Sigma. Columns for FPLC were purchased from Pharmacia. $\mathrm{H}_{2} \mathrm{O}_{2}(30 \% \mathrm{w} / \mathrm{v})$ was from Merck.

Large-scale protein purification. Spores of Streptomyces reticuli were added to basal minimal medium supplemented with $1 \%$ crystalline cellulose (Avicel TG 104, Sigma) in baffled 11 Erlenmeyer flasks for $2 \mathrm{~d}$. Streptomyces reticuli was grown in minimal medium supplemented with $1 \%$ Avicel in 10 aliquots of $700 \mathrm{ml}$, as described by Schlochtermeier et al. (1992b). Mycelia were harvested by centrifugation (20 min at $100000 \mathrm{~g}$ ) and washed three times with $20 \mathrm{mM}$ potassium phosphate buffer, $(\mathrm{pH} 7) / 0 \cdot 1 \%$ Triton X-100. The combined wash fractions $(2 \cdot 21)$ were loaded onto a DEAE column (Pharmacia 16/10) previously equilibrated in $20 \mathrm{mM}$ sodium phosphate buffer ( $\mathrm{pH} 7 \cdot 0$ ), supplemented with $0.05 \%$ Triton $\mathrm{X}-100$ and eluted with a $90 \mathrm{ml}$ gradient $(0-0.5 \mathrm{M} \mathrm{NaCl})$. The catalase-peroxidase-containing fractions eluted at $0.25-0.3 \mathrm{M}$ $\mathrm{NaCl}$. The samples from the DEAE column were diluted with phosphate buffer and loaded onto a MonoQ (5/5) column. Proteins were eluted with a $50 \mathrm{ml}$ gradient $(0-0.5 \mathrm{M} \mathrm{NaCl})$ at a flow rate of $1 \mathrm{ml} \mathrm{min}{ }^{-1}$ with the buffer described above. The proteins eluted in the presence of $0.3-0.35 \mathrm{M} \mathrm{NaCl}$ displayed catalase and peroxidase activity. These fractions were pooled, dialysed against phosphate buffer with $150 \mathrm{mM} \mathrm{NaCl}$ and loaded onto a Superose 6 (HR10/30) column (Pharmacia), equilibrated with $20 \mathrm{mM}$ sodium phosphate buffer containing $150 \mathrm{mM} \mathrm{NaCl}$. The protein concentration of the samples was determined employing the method of Bradford (1976) and the Lowry method.

Production of antibodies. Antibodies were raised against the purified catalase-peroxidase. Antisera from rabbits were obtained by immunization with the lyophilized enzymes mixed with Freund's complete and incomplete adjuvants. The rabbits were bled $10 \mathrm{~d}$ after the third booster injection. Cellular particles were removed by centrifugation and the antisera were stored in aliquots at $-20^{\circ} \mathrm{C}$.

Determination of $\mathbf{N}$-terminal amino acids. The purified mature enzyme and peptides (generated by the LysC protease) were commercially determined by Edman degradation (Wita).

Native and SDSPAGE. For electrophoresis of the native enzyme, a $10 \%$ (w/w) polyacrylamide gel, $\mathrm{pH} 7 \cdot 5$, was used (Laemmli, 1970). SDS-PAGE was performed in the presence of $0 \cdot 1 \%$ SDS (Laemmli, 1970).

Detection of catalase activity. The native polyacrylamide gel was washed three times for $15 \mathrm{~min}$ with distilled $\mathrm{H}_{2} \mathrm{O}$, suspended in a solution of $0.01 \mathrm{ml} 30 \% \mathrm{H}_{2} \mathrm{O}_{2}$ in $100 \mathrm{ml} \mathrm{H}_{2} \mathrm{O}$ and gently rocked for $10 \mathrm{~min} . \mathrm{H}_{2} \mathrm{O}_{2}$ was aspirated and the gel quickly rinsed in $\mathrm{H}_{2} \mathrm{O}$. A freshly prepared mixture of $30 \mathrm{ml}$ each of $2 \%$ ferric chloride and $2 \%$ potassium ferricyanide, both in $\mathrm{H}_{2} \mathrm{O}$, was poured into a fresh staining pan and the rinsed gel transferred to the ferricyanide mixture (Wayne $\&$ Diaz, 1986). The gel tray was gently but steadily rocked by hand over a light box. As soon as a green colour began to appear in the gel itself, the ferricyanide mixture was rapidly aspirated and replaced with water. The gel was washed twice with water.

Test for peroxidase activity. Samples were loaded onto a 
native $10 \%$ polyacrylamide gel. After the run, the gel was washed twice with acetate buffer $(20 \mathrm{mM}, \mathrm{pH} 5.5)$ and activity staining was carried out with $4 \mathrm{C} 1 \mathrm{~N}$ and $5 \mathrm{mM} \mathrm{H}_{2} \mathrm{O}_{2}$ (Conyers $\&$ Kidwell, 1991). The peroxidase activity was monitored spectrophotometrically at room temperature in a reaction mixture containing $50 \mathrm{mM}$ sodium acetate buffer $(\mathrm{pH} \mathrm{5.5)}$, $2 \mathrm{mM} \mathrm{H}_{2} \mathrm{O}_{2}$ and $1 \mathrm{mM} \mathrm{O}$-dianisidine by following the rate of oxidation at $460 \mathrm{nM}\left(\varepsilon_{460} 11.3 \times 10^{3} \mathrm{M} \mathrm{cm}^{-1}\right)$. One unit of peroxidase activity is defined as the amount of enzyme causing the disappearance of $1 \mu \mathrm{mol}$ substrate $\min ^{-1}$.

Isolation of total DNA and plasmids. Chromosomal DNA of Streptomyces reticuli was isolated after growth in a sucrosecontaining complete medium for $2 \mathrm{~d}$ (Dittrich et al., 1991). The E. coli plasmid pUC18 was isolated using the alkaline lysis method (Sambrook et al., 1989) and the multicopy Streptomyces/E. coli vector pWHM3 was obtained by a modified alkaline lysis method (Dittrich et al., 1991; Hopwood et al., 1985).

Cleavage of DNA, ligation and agarose gel electrophoresis. The DNAs were cleaved with various restriction enzymes according to the suppliers' instructions. Ligation was performed with T4 ligase (Sambrook et al., 1989). Gel electrophoresis was carried out in $0.8-1 \%$ agarose gels using TBE buffer. Fragments were visualized under UV after staining with ethidium bromide (Sambrook et al., 1989).

Transformations. E. coli was transformed with plasmid DNA using the $\mathrm{CaCl}_{2}$ method (Sambrook et al., 1989) or by electroporation (Dower et al., 1988). Streptomyces lividans 66 protoplasts were transformed and regenerated as described by Hopwood et al. (1985). Transformants were selected using an overlay of $0.4 \%$ agarose containing $500 \mu \mathrm{g}$ thiostrepton $\mathrm{ml}^{-1}$ (Hopwood et al., 1985).

Screening of a $\lambda$ gt11 gene library. In our laboratory, a library of genomic DNA fragments (in the range $0.4-4.0 \mathrm{~kb}$ ) was constructed in the bacteriophage expression vector igt11 (Schlochtermeier et al., 1992b). Phages were propagated in $E$. coli Y 1090 and screened with antibodies. Filters were incubated in PBS containing a $1: 10^{5}$ dilution of the anti-CpeB antibodies and washed three times in PBS. Then they were incubated with alkaline-phosphatase-conjugated goat antirabbit $\lg \mathrm{F} F(\mathrm{ab})_{2}$, diluted 1:30000 in PBS, followed by colour development (West et al., 1990). Immunopositive plaques were collected and rescreened to purity.

Hybridization experiments. The transfer of DNA fragments of a subgenomic Streptomyces reticuli library from Bam $\mathrm{HI}$ fragments $(6-8 \mathrm{~kb})$ and a Streptomyces reticuli genomic library in $\lambda$ Charon 35 (Schlochtermeier et al., 1992b) onto nylon membranes was performed as described by Sambrook et al. (1989). DNA from phage plaques was transferred to nitrocellulose filters (Allday \& Jones, 1987). The inserts isolated from hybrid $\lambda$ gt 11 phages were labelled using Klenow enzyme and digoxigenin-11-dUTP. Hybridization and immunological detection were carried out according to the specifications of the DNA Labelling and Detection Kit supplied by Boehringer Mannheim.

DNA sequencing and computer analysis. For sequencing, specific DNA fragments were subcloned into pUC18/19. Overlapping deletions were generated by exonuclease III digestion (Henikoff, 1987). Non-radioactive sequencing was performed with the GATC kit (Beck \& Pohl, 1984), using standard primers of the lac $Z$ system as well as synthesized primers. Sequence entry, primary analysis and ORF searches were performed using CloneManager 5.0. Database searches using the PAM120 scoring matrix were carried out with BLAST algorithms (BLASTX, BLASTP and TBLASTN) on the NCBI file server (blast@ncbi.nlm.nih.gov) (Altschul et al., 1997). Multiple sequence alignments were generated by means of the CLUSTAL W (1.74) program (Higgins et al., 1992).

Putative Shine-Dalgarno (ribosome-binding) sites (Gold et al., 1981; Strohl, 1992) and signal peptide cleavage sites (Nielsen et al., 1997) were predicted as described.

\section{RESULTS}

\section{Purification of a mycelium-associated Streptomyces reticuli catalase-peroxidase}

When studying the cellulolytic system of Streptomyces reticuli, we noticed that the strain produces a myceliumassociated catalase-peroxidase in addition to the previously described mycelium-associated cellulase 1 (Avicelase) (Schlochtermeier et al., 1992b). The isolation procedure was optimized and larger amounts of the protein were purified. To achieve this, proteins were released from the mycelia by three consecutive washings in buffer supplemented with $0 \cdot 1 \%$ Triton X100 and were separated by anion exchange chromatography (DEAE and MonoQ), followed by Superose 6. The size of the enzymes was determined to be 160 and $82 \mathrm{kDa}$, respectively, by gel filtration and SDS-PAGE (Fig. 1). Thus the native $\mathrm{CpeB}$ consists of two identical subunits. Spectroscopical investigations revealed that a solution of the enzyme has a pronounced Soret band at $406 \mathrm{~nm}$ and two smaller peaks at 503 and $643 \mathrm{~nm}$. The addition of $\mathrm{KCN}(10 \mathrm{mM})$ resulted in a shift of the Soret band to $421 \mathrm{~nm}$, the appearance of a peak at $540 \mathrm{~nm}$ and of a shoulder at $637 \mathrm{~nm}$. The ratio $A_{406}: A_{280}$ for the pure enzyme was 0.525 , suggesting that one molecule of protohaem IX was present per $\mathrm{CpeB}$ dimer. The enzyme acts as catalase-peroxidase and accepts a broad range of substrates, hence the designation $\mathrm{CpeB}$, including ABTS, benzidine, 3-dimethylaminobenzoic acid, 3-methyl-2- (a)

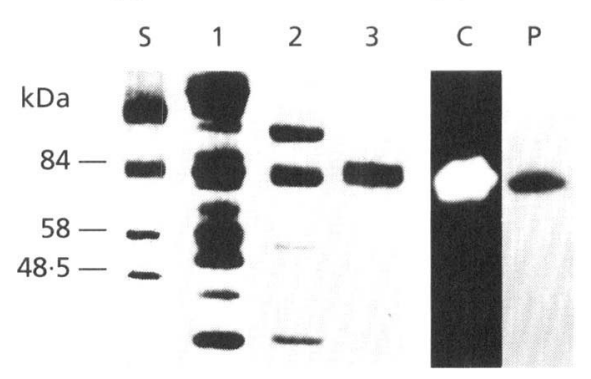

Fig. 1. Purification of the enzyme and its analysis. (a) Streptomyces reticuli was cultivated as described in Methods. Aliquots of the total proteins released from the mycelia (lane 1), of a fraction from the DEAE column (lane 2) and of the Superose column (lane 3 ), as well as reference proteins (lane S) were subjected to SDS-PAGE and proteins were stained by Coomassie blue. (b) An aliquot of the purified Streptomyces reticuli enzyme CpeB (presented in lane 3 ) was loaded onto a native polyacrylamide gel. After the run, enzymic tests were performed as outlined in Methods. Lanes: $C$, test for catalase activity; $P$, test for peroxidase activity. 


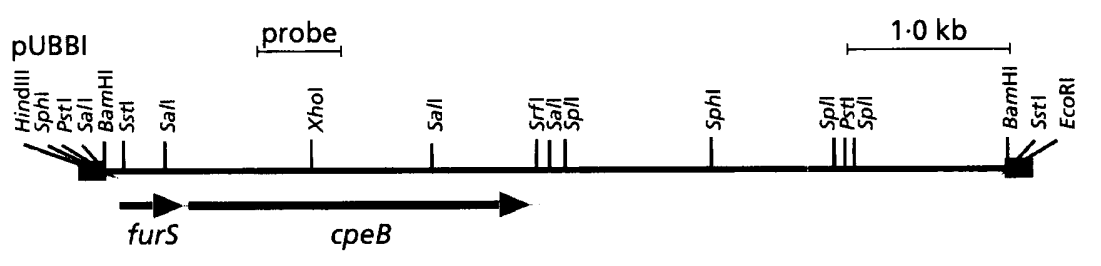

pUKS10
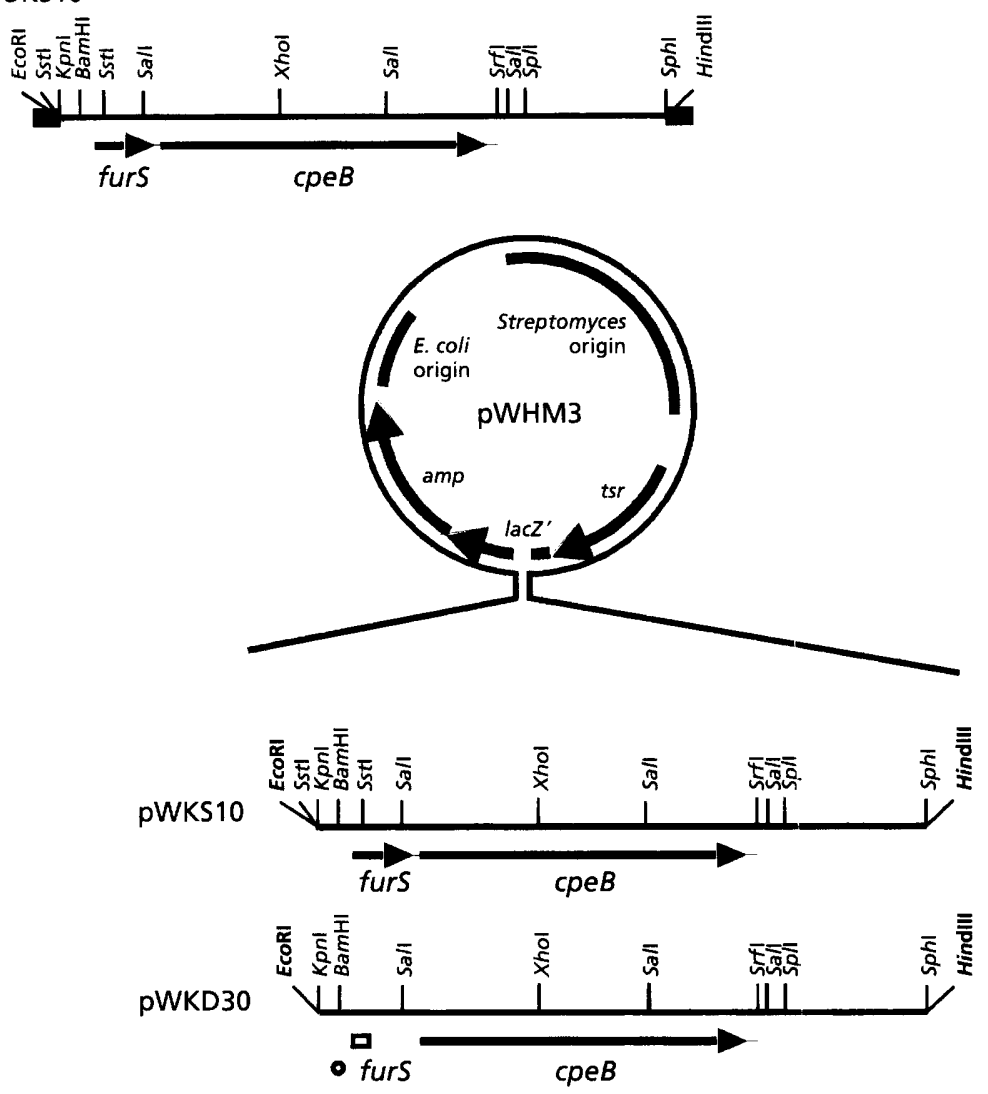

Fig. 2. Restriction maps of constructs. Cloning of the $6 \mathrm{~kb}$ Streptomyces reticuli BamHI fragment and of the 4.6 Kpnl-Sphl fragment in pUC18 resulted in the formation of PUBB1 and pUKS10, respectively. Recloning of the Kpnl-HindIII fragment into the bifunctional $E$. colil Streptomyces vector pWHM3 (Vara et al., 1989) led to the formation of pWKS10. Recloning of the Kpnl-HindIII fragment of pUKD30 (carrying an Sstl deletion in the polylinker of the vector and the insert) led to plasmid pWKD30.

benzothiazolinone hydrazone (MBTH), 3,3'-diaminobenzidine, 4C1N, NADH, NADPH, ascorbate, guaiaco] and veratrylalcohol.

\section{Screening of an expression library and cloning of the gene}

Five hundred micrograms of the highly purified $82 \mathrm{kDa}$ enzyme was used to raise antibodies. In addition, the Nterminal amino acids from the LysC protease-generated internal peptides of the purified enzyme were determined by Edman degradation. The antibodies and a previously constructed expression library (in igt11) of Streptomyces reticuli total DNA (Schlochtermeier et al., 1992b) were used to screen for hybrid phages. Two phages synthesized peptides cross-reacting with anti-CpeB antibodies. The phage DNAs were isolated and their inserts $(0.5$ and $0.7 \mathrm{~kb}$ fragments carrying the EcoRI linkers of $\lambda$ gt11) were sequenced. An analysis of the possible ORFs allowed the deduction of a stretch of amino acids, which shares a high degree of similarity to a block of amino acids, including the proximal histidine ligand involved in haem binding within bacterial catalase-peroxidases.
The $0.5 \mathrm{~kb}$ fragment hybridized to a $6 \mathrm{~kb}$ Bam HI fragment, a $1.8 \mathrm{~kb}$ Sall fragment and a $4.6 \mathrm{~kb} K p n \mathrm{I}-S p h \mathrm{I}$ fragment from total Streptomyces reticuli DNA (Fig. 2). Subgenomic libraries of Bam HI fragments $(5-7 \mathrm{~kb})$ and $K p n \mathrm{I}-S p h \mathrm{I}$ fragments $(4-5 \mathrm{~kb})$ from Streptomyces reticuli DNA were constructed with pUC18. Ampicillinresistant E. coli JM83 transformants were screened by colony hybridization. Several clones carrying the correct insert were identified. The $6 \mathrm{~kb} \mathrm{BamHI}$ insert of one of them, pUBB1, was characterized by restriction enzyme analysis (Fig. 2). The insert of pUKS10 containing a $4.6 \mathrm{~kb} K p n \mathrm{I}-S p h \mathrm{I}$ fragment was found to overlap with the main part of the Bam HI insert and to stretch beyond one of its ends.

\section{Determination of the sequence and its analysis}

The sequence of $3193 \mathrm{bp}$ corresponding to a KpnI-SrfI fragment was determined. Two potential ORFs were found. A small one (furS) starts with a start codon (ATG) at position 385 and ends with a stop codon (TGA) at position 822. The larger ORF ( $c p e B)$ comprises 


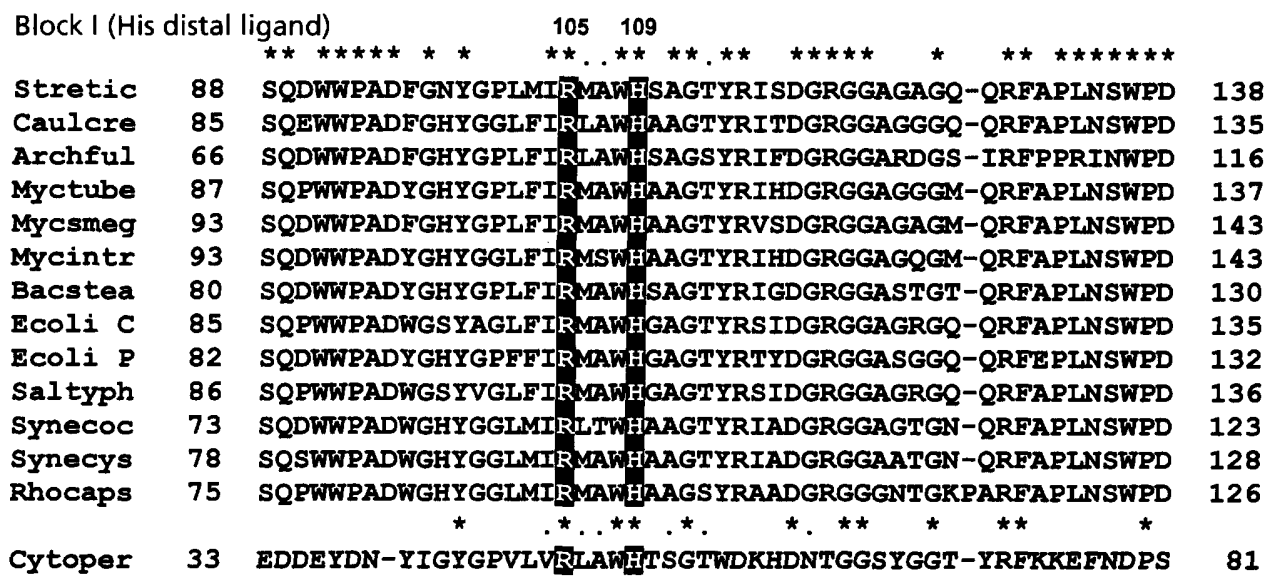

\begin{tabular}{|c|c|c|c|c|}
\hline \multicolumn{5}{|c|}{ Block II (His proximal ligand) } \\
\hline & & 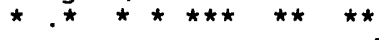 & 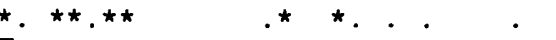 & \\
\hline ic & 251 & DEE? & DHVGADPEAAS & 301 \\
\hline$r e$ & 24 & NDEETVAI & ASLVGVEPEGGAIEAOGGF & \\
\hline ul & 22 & IFRRMGMNDEETVA & KCHGAG-PADIYGPDPS & \\
\hline be & 4 & GTFRRMAMNDVETAALI & KTHGAG-PADLVGPE PEAAPLEQMGLG & 95 \\
\hline$e q$ & 5 & RMAMNDVETAALI & KTHGNG-DASLVGPEPE & \\
\hline & $256+2>+>$ & RMAMNDEETAAI & KTHGAG-DADLVGPEPI & \\
\hline & 24 & NDEETVA & HHGAG-PATHVGPEPEAAPI & \\
\hline zoli C & 24 & FGNMGMNDEETVA & IKTHGAG-PTSNVGPDPE & \\
\hline $\mathbf{P}$ & 24. & DDEETVAI & AHGAAS PEKCIGAGPL & \\
\hline ph & 247 & FGNMGMNDEETVAI & -AASHVGADPE? & \\
\hline oc & 243 & ANDEETVA & -NAALLGPEPE & \\
\hline & 244 & TTEARMAMNDEETVA & -KAELIGPEPEC & \\
\hline ps & 244 & RETEARMGMNDEETVA & ALGPDPEAAD & \\
\hline & & MNDREVVAUM- & IKNSGYEGPWGAAN & \\
\hline
\end{tabular}

Fig. 3. Comparisons of block motifs. Blocks I and II from the deduced Streptomyces reticuli catalase-peroxidase (Stretic) containing both the distal and the proximal histidine ligand (black) were aligned with the corresponding regions of deduced catalase-peroxidases from: Caulobacter crescentus, Caulcre (AF027168); Archaeoglobus fulgidus, Archful (AE000591); Mycobacterium tuberculosis, Myctube (X68081); Mycobacterium smegmatis, Mycsmeg (X98718); Mycobacterium intracellulare, Mycintr (M86741); Bacillus stearothermophilus, Bacstea (M29876); E. coli (chromosomal) Ecoli C (M21516) and E. coli (plasmid) Ecoli P (X89017); Salmonella typhimurium, Saltyph (X53001); Synechococcus sp. PCC 7942, Synecoc (D61378); Synechocystis sp. PCC 6803, Synecys (D90910); Rhodobacter capsulatus, Rhocaps (X71420); and the cytochrome $c$ peroxidase from Saccharomyces cerevisiae, Cytoper (X62422; aa numbering from aa 68 of the precursor). All sequences were deduced from sequences deposited in GenBank. Identical $(*)$ and similar (.) amino acid residues are marked. The additional lane of '*' and ' $\because$ ' at the bottom shows identical and similar amino acids between the alignment of bacterial KatG sequences and the cytochrome $c$ peroxidase sequence.

a start codon (ATG) at position 856 and a stop codon (TGA) at position 3078. The G $+\mathrm{C}$ content of $c p e B$ is $69.6 \mathrm{~mol} \%$ and that of furS is $73.5 \mathrm{~mol} \%$.

\section{The deduced proteins}

$c p e B$ encodes a protein of 740 aa with a predicted molecular mass of $81346 \mathrm{Da}$, corresponding to that of the purified monomeric form (apparent molecular mass $82 \mathrm{kDa}$ ) of the Streptomyces reticuli enzyme (Fig. 1). The calculated isoelectric point is $4 \cdot 88$. The experimentally determined amino acid sequence of the $\mathrm{N}$ termini of the internal peptides corresponded to that in the protein deduced from the DNA sequence. No predictable signal peptide could be identified in the deduced $\mathrm{CpeB}$. Within several regions, the deduced protein shares a high number of identical amino acids with several other deduced bacterial catalase-peroxidases (Fig. 3) and includes the regions containing the amino acid residues essential for the catalytic centre ('distal' His109) and for haem-binding ('proximal' His272). The number of identical amino acids ranges from 63 to $76 \%$ (block I) and from 39 to $72 \%$ (block II), depending on the origin of the corresponding enzyme (Fig. 3 and Discussion). Moreover, the relative positions of the amino acid residues Arg105, His109 and His272 correspond to those of the yeast cytochrome $c$ peroxidase (Fig. 3). These residues are essential for the formation of the catalytic centre within other haemcontaining peroxidases (Smulevich, 1995; Poulos et al., 1995).

The deduced protein (FurS) encoded by the small ORF 
(a)

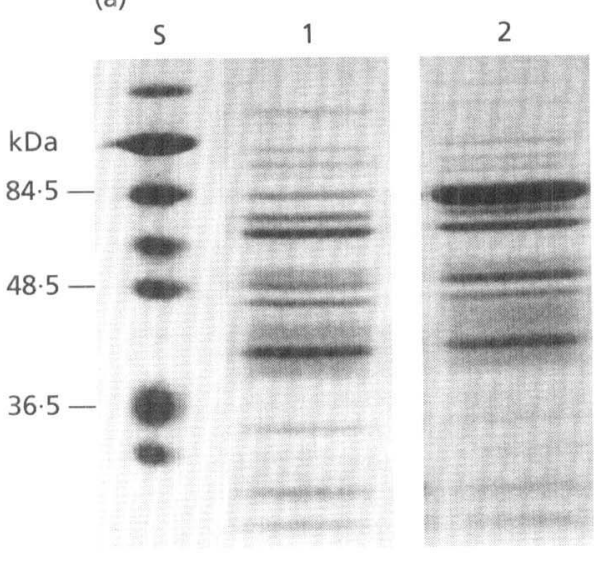

(b)

\section{2}

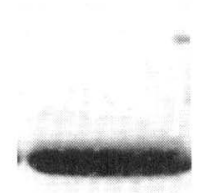

Fig. 4. Analysis of Streptomyces lividans transformants. (a) Streptomyces lividans(pWHM3) (control, lane 1) and Streptomyces lividans(pWKS10) (lane 2) were cultivated as outlined for Streptomyces reticuli (Fig. 1) for 72 h. Proteins were released from the mycelia and, together with reference proteins (lane S), subjected to SDS-PAGE and either stained with Coomassie blue or transferred to a nylon membrane. (b) The proteins were inspected as to their cross-reaction with anti-CpeB antibodies. (c) Samples were analysed for peroxidase activity on native gels. (d) The mycelium-associated enzyme from Streptomyces lividans(pWKS10) was purified as described for Streptomyces reticuli (Fig. 1) and tested for catalase (lane C) and peroxidase (lane $\mathrm{P}$ ) activity on native gels. In addition, one sample was stained with Coomassie blue (lane $\mathrm{Co}$ ). comprises $145 \mathrm{aa}$; it has a molecular mass of $15123 \mathrm{Da}$ and a calculated pI of $5 \cdot 35$. FurS shares $29 \%$ identical and $44 \%$ similar amino acids with the E. coli Fur (ferric uptake regulator) protein. The relative location of the His91/His93 and Cys96/Cys99 residues of FurS corresponded to that of the predicted metal-binding sites (His88/His90 and Cys93/Cys96) of the deduced E. coli Fur protein (Bagg \& Neilands, 1987).

The multiple sequence alignment analysis of more than 45 known Fur and Fur-like proteins (used to generate the phylogenetic tree in Fig. 7) showed that Tyr59 (aa numbering for FurS) is completely conserved (alignment not shown).

\section{Expression of the enzyme in Streptomyces lividans}

The $4.6 \mathrm{~kb} K p n \mathrm{I}-S p h \mathrm{I}$ fragment from pUKS10 was cloned into pWHM3, inducing the formation of pWKS10, which in turn was cloned into Streptomyces lividans as host. Like Streptomyces reticuli, the Streptomyces lividans transformant carrying pWKS10 produced a mycelium-associated catalase-peroxidase that could be reproducibly released by washing the mycelia with buffer supplemented with Triton X100. Moreover. antibodies raised against the enzyme purified from. Streptomyces reticuli cross-reacted with one of the proteins released from the mycelia of Streptomyces: lividans(pWKS10). However, no cross-reacting protein was detected among the proteins released from the control strain Streptomyces lividans(pWHM3) (Fig. 4). In conclusion, the data show that the cloned gene encoded the enzyme characterized from Streptomyces: reticuli (Fig. 1).

\section{Regulation}

Secondary structure predictions suggest that the deduced protein FurS contains a putative HTH (helix-turnhelix) motif within the N-terminal region (Fig. 5b). Therefore, we concluded that it may represent a regulatory DNA-binding protein. To test whether the upstream gene affects expression of $c p e B$, the KpnIHindIII insert of pWKS10 was cloned into pUDS18 (a derivative of pUC18 carrying an SstI deletion). The Sst I site of the resulting construct was removed (after cleavage, Klenow treatment and religation in plasmid pUKD30). By sequencing it could be shown that plasmid DNA of several transformants containing pUKD30 carry the SstI site deletion. The deduced truncated protein contains only 11 of the $\mathrm{N}$-terminal amino acids of FurS. The KpnI-HindIII fragment of pUKD30 containing the in-frame deletion was then recloned in the bifunctional vector pWHM3 (Fig. 2), entailing the formation of $\mathrm{pWKD} 30$. The level of peroxidase activity was compared in Streptomyces lividans transformants containing either $\mathrm{pWKS10}$ or $\mathrm{pWKD} 30$. In the presence of $\mathrm{Fe}$ ions, the peroxidase activity of Streptomyces lividans(pWKD30) was several times higher than that of Streptomyces lividans(pWKS10). Immunological tests and analyses of protein-stained gels showed that Streptomyces lividans (pWKS10) produced low levels of the enzyme in the presence of $\mathrm{Fe}$ ions and enhanced levels in the presence of chelators for $\mathrm{Fe}^{3+}$ and $\mathrm{Fe}^{2+}$ ions, respectively (diethylenetriaminepentaacetic acid and EDTA). Streptomyces lividans(pWKD30), on the other hand, produced higher quantities of CpeB than Streptomyces lividans(pWKS10) and this amount was not affected by the presence of $\mathrm{Fe}^{3+/ 2+}$ chelators (Fig. 6). The 
(a)

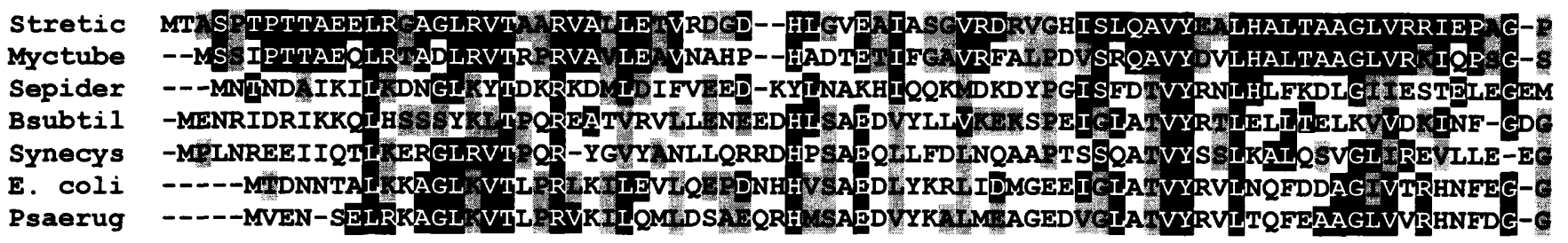

Stretic

Myctube

Sepider

Bsubtil

Synecys

E. coli

Psaerug

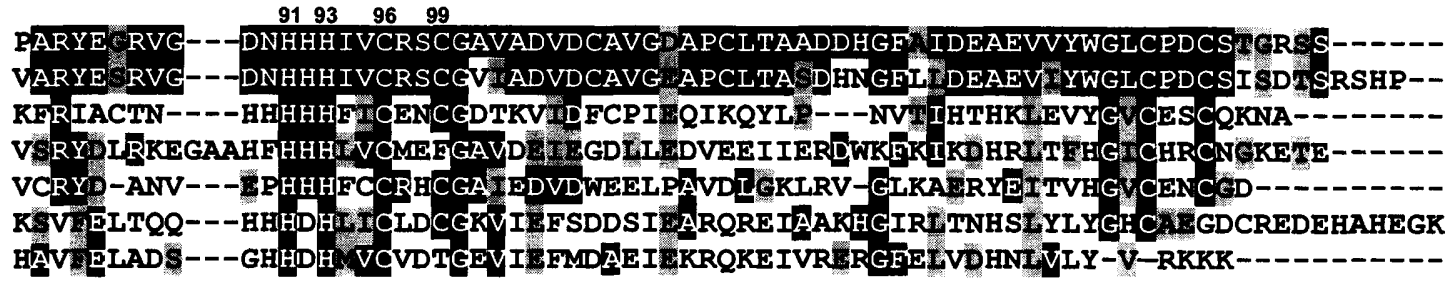

(b)

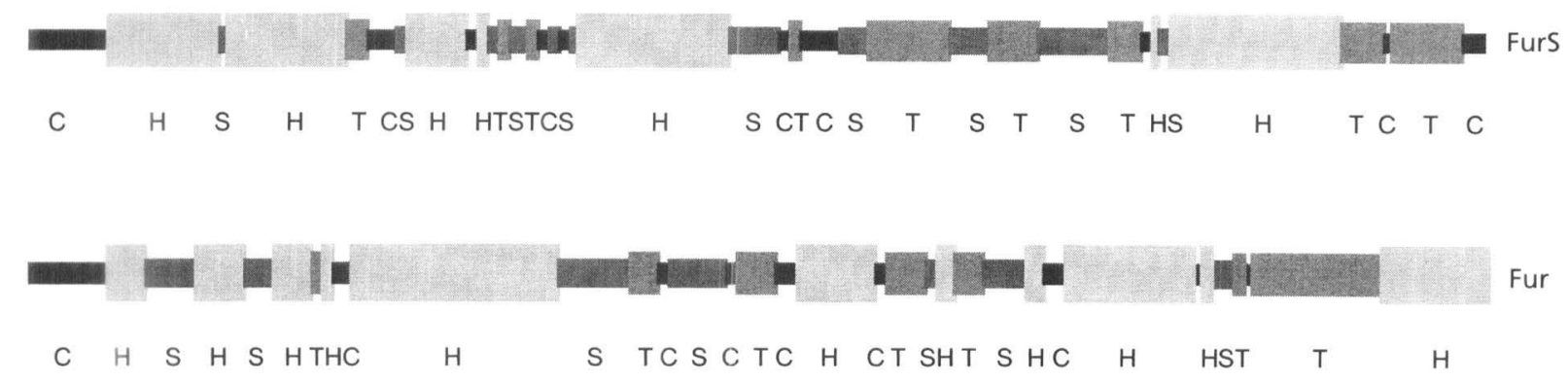

Fig. 5. Comparison of FurS and Fur proteins. (a) Deduced amino acid sequence of Streptomyces reticuli (Stretic) furS aligned with Fur and Fur-like proteins deduced from sequences deposited in GenBank: Mycobacterium tuberculosis, Myctube (X68081); Staphylococcus epidermidis, Sepider (X97011); Bacillus subtilis, Bsubtil (D84432); Synechocystis sp. PCC 6803, Synecys (D90909); E. coli, E. coli (X02589); Pseudomonas aeruginosa, Psaerug (L00604). (b) Secondary structure prediction. Comparison of predicted secondary structures of FurS (Streptomyces reticuli) and Fur $(E$. coli) proteins; four secondary structural elements, coils $(C), \alpha$-helices $(H), \beta$-sheets $(S)$ and turns $(T)$ are shown as differently shaded boxes.

data imply that FurS is a repressor and regulates the transcription of $c p e B$.

\section{DISCUSSION}

During the early stages of growth, Streptomyces reticuli was found to synthesize a mycelium-associated dimeric catalase-peroxidase with broad substrate specificity. After its purification, the corresponding gene was characterized. An analysis of the deduced $\mathrm{CpeB}$ protein $(81 \cdot 3 \mathrm{kDa})$ revealed that it shares a high degree of amino acid identity with enzymes of similar size, including KatG from Caulobacter crescentus (Steinman et al., 1997), Mycobacterium tuberculosis (Zhang et al., 1992; Nagy et al., 1995) and Mycobacterium smegmatis (Marcinkeviciene et al., 1995; Magliozzo \& Marcin- keviciene, 1997), KatP from E. coli (Brunder et al., 1996), and PerA from Archaeoglobus fulgidus (GenBank AE000951) and Bacillus stearothermophilus (Loprasert et al., 1988) (Fig. 3). The unrooted phylogenetic tree (data not shown) confirms that $\mathrm{CpeB}$ belongs to the plant type of peroxidases. Subfamily 1 , to which it belongs, contains yeast cytochrome $c$ peroxidase, ascorbate peroxidase and bacterial catalase-peroxidases (Welinder, 1991). Key residues required for catalytic activities are highly conserved among all members of the catalase-peroxidases. Gapped multiple sequence alignments (data not shown) disclosed variations in the length of amino acid 'stretches' separating the distal and the proximal histidine residues. Only the introduction of a gap of 37-39 aa leads to an alignment of these enzymes with haem-dependent peroxidases. This variability may explain the substrate plasticity of catalase-peroxidases, as compared to monofunctional 
(a)

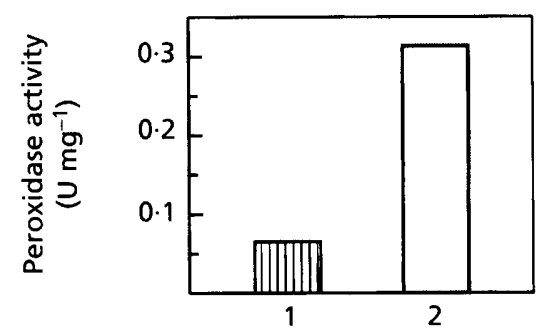

(b)

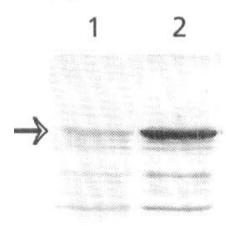

(d)

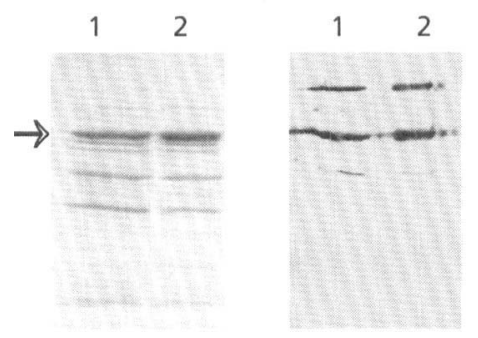

Fig. 6. Regulation of enzyme synthesis. Streptomyces lividans(pWKS10) (column/lanes 1) and Streptomyces lividans(pWKD30) (column/lanes 2) were cultivated for $40 \mathrm{~h}$ and shifted to medium containing $5 \mu \mathrm{M}$ Fe ions for $30 \mathrm{~h}$. Aliquots of released mycelium-associated proteins were tested for peroxidase activity (a), analysed on SDS-PAGE gels stained with Coomassie blue (b) or were tested with anti-CpeB antibodies after transfer of the SDS-gel to a membrane (c). In addition, each strain was shifted to a medium containing the chelator for $\mathrm{Fe}^{3+}$ and $\mathrm{Fe}^{2+}$ ions (diethylenetriaminepentaacetic acid and EDTA). The proteins released from the mycelia were analysed on SDS-PAGE gels stained with Coomassie blue (d) or with anti-CpeB antibodies after transfer (e).

peroxidases. In this context, it is interesting that the Streptomyces reticuli $\mathrm{CpeB}$ enzyme, unlike all other tested catalase-peroxidases, also oxidizes ascorbate, guaiacol and veratrylalcohol. All known catalaseperoxidases studied to date, except KatP, have been found within the cytoplasm. The Streptomyces reticuli $\mathrm{CpeB}$ enzyme, in contrast, is extracellularly loosely associated with the mycelia. As we could not predict a corresponding signal peptide on the basis of known signal peptide sequences of bacteria, we will investigate whether $\mathrm{CpeB}$ may be exported by a sec-independent secretion machinery which has been identified in other bacteria (Wattiau et al., 1996; Pugsley et al., 1991). E. coli KatP, on the other hand, has a predicted signal peptide and was detected within the periplasm (Brunder et al., 1996).

Our data revealed that the synthesis of the Streptomyces reticuli catalase-peroxidase is under the control of a regulatory gene, whose gene product FurS shares $29 \%$ identical and $44 \%$ similar amino acids with the $E$. coli Fur protein. Homologues of E. coli Fur have been characterized predominantly in various Gram-negative bacteria (Bourke et al., 1996; Hantke, 1984). Recently, fur-like genes have also been reported in three Grampositive bacteria: Staphylococcus epidermidis (Heidrich et al., 1996), Bacillus subtilis (GenBank Z82044, D84432) and Streptococcus pyogenes (GenBank U76538). Fur proteins are rich in histidine and cysteine residues and contain an N-terminally located DNA-binding region, as well as a C-terminal domain binding metal ions $\left(\mathrm{Fe}^{2+}\right.$ or other divalent first row transition elements) (Bagg \& Neilands, 1987). Upon binding of the metal ions to the C-terminal domain, a conformational change is induced within the N-terminal part of the protein, leading subsequently to the interaction of the Fur homodimer with the corresponding operator sequence. When complexed with $\mathrm{Fe}^{2+}$, E. coli Fur and its close bacterial homologues act as repressors of a number of genes (Ochsner \& Vasil, 1996; Hantke, 1984, 1981). Recent studies with Salmonella typhimurium have indicated that the global regulatory protein Fur can regulate gene expression both in a positive and in a negative way and that regulation also occurs in the absence of the ironsensing function of Fur (Hall \& Foster, 1996).

When grown in the presence of varying concentrations of Fe ions, Streptomyces lividans(pWKS10), containing the intact furS gene, synthesized low levels of CpeB. However, if the strain is cultivated with compounds chelating $\mathrm{Fe}^{2+}$ or $\mathrm{Fe}^{3+}$ ions, higher amounts of $\mathrm{CpeB}$ protein are synthesized, as shown by immunological studies. In contrast, Streptomyces lividans(pWKD30), carrying an in-frame deletion in furS, produced enhanced levels of $\mathrm{CpeB}$ protein. These data suggest that in the presence of Fe ions, FurS acts as repressor of $c p e B$. OxyR regulates the transcription of E. coli kat $G$ (encoding hydroperoxidase I) (Storz et al., 1990; Zheng et al., 1998). Within Mycobacterium tuberculosis, however, oxyR is inactivated by multiple lesions. These findings refuted the initial assumption that the transcription of the mycobacterial kat $G$ gene is under the control of an OxyR-type regulator (Deretic et al., 1995).

In the course of sequencing projects, several furA genes could be identified within different mycobacteria, the deduced proteins of which are closely related to Streptomyces reticuli FurS. FurS and FurA (Mycobacterium tuberculosis) show $64 \%$ identity and $77 \%$ similarity; the internal FurS region (Gln56 to Ser140) even shares $80 \%$ identical and $94 \%$ similar amino acids with the corresponding part of the Mycobacterium tuberculosis homologue (Fig. 5a). It is intriguing that all kat $G$ genes found within the genomes of different mycobacteria contain an upstream-located furA gene (Fig. 7). Moreover, a recently sequenced genomic region within Mycobacterium leprae, which is linked to a non- 


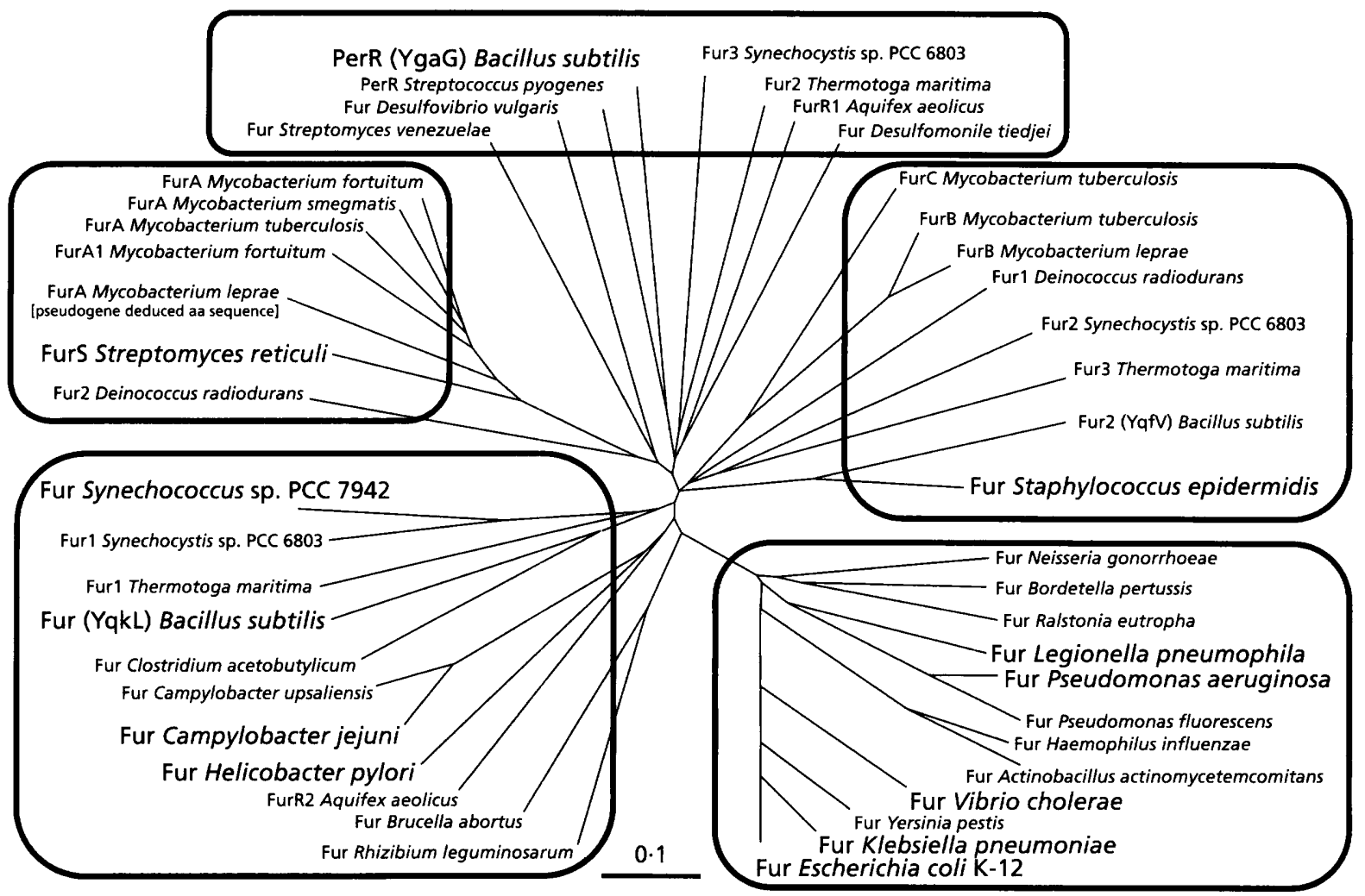

Fig. 7. Unrooted phylogenetic tree of the deduced amino acid sequences of bacterial fur and fur-like genes. All sequences used were obtained from the GenBank database [Mycobacterium tuberculosis FurA, AF002194; Mycobacterium leprae pseudo-FurA, AF13983; Mycobacterium smegmatis FurA, AF012631; Mycobacterium fortuitum FurA1, Y07865*. Mycobacterium fortuitum, FurA2, Y07866*; Mycobacterium tuberculosis FurB, Z95208; Mycobacterium leprae FurB, L78812*; Streptomyces venezuelae, X74791; Desulfovibrio vulgaris, U82323; E. coli K-12, X02589; Klebsiella pneumoniae, L23871; Yersinia pestis, S47625; Vibrio cholerae, M86629; Haemophilus influenzae, L42023; Neisseria gonorrhoeae, L11361; Bordetella pertussis, L31851; Pseudomonas aeruginosa, L00604; Pseudomonas fluorescens, AF050677; Legionella pneumophila, U06072; Ralstonia eutropha, AJ001224; Campylobacter jejuni, Z35165; Campylobacter upsaliensis, L77075; Synechococcus sp. PCC 7942, L41065; Synechocystis sp. PCC 6803 Fur1, D90909; Synechocystis sp. PCC 6803 Fur2, D90903; Synechocystis sp. PCC 6803 Fur3, D90917; Staphylococcus epidermidis, X97011; Bacillus subtilis Fur (YqkL), D84432; Bacillus subtilis Fur2 (YqfV), Z99116; Bacillus subtilis PerR (YgaG), Z99108; Desulfomonile tiedjei, AF015192*; Aquifex aeolicus FurR1, AE000728; Aquifex aeolicus FurR2, AE000739; Brucella abortus, AF023177; Rhizobium leguminosarum, Y13657; Helicobacter pylori, AE000611; Streptococcus pyogenes PerR (Fur) U76538], the TIGR database [contig gmt7891 encoding the FurC protein (database for the Mycobacterium tuberculosis clinical isolate \#CSU94 genome), contigs gdr_186 and gdr_157 encoding Fur1 and Fur2, respectively, in the Deinococcus radiodurans genome; contigs BTMCU54R, BTMDR61R and BTMDH20F encoding Fur1, Fur2 and Fur3, respectively, in the Thermotoga maritima genome], the Oklahoma University (OU_ACGT) database [contig 874 (Actinobacillus actinomycetemcomitans genome)] and the Genome Therapeutics Corp. database [contig 112 (Clostridium acetobutylicum ATCC 824 genome)]. An asterisk next to the above accession numbers indicates the presence of a fur-like gene that has not been annotated by the authors. The CLUSTAL w (1.74) software on the EMBL file server (http://www2.ebi.ac.uk) was used for multiple sequence alignments and computation of the phylogenetic tree. The scale represents 0.1 aa changes per position. The Fur and Fur-like proteins (and the corresponding bacteria) whose properties have been elucidated are printed in larger type.

functional $k a t G$ gene, represents a furA-like pseudogene (Eiglmeier et al., 1997; Nakata et al., 1997). Using primers deduced from the genomic Mycobacterium tuberculosis sequence, a homologous furA-kat $G$ locus was cloned from the non-tuberculous pathogen $\mathrm{Myco-}$ bacterium marinum (Pagan-Ramos et al., 1998).

In contrast, the upstream regions of catalase-peroxidase genes sequenced to date from various other bacteria do not contain fur or fur-like genes (Fig. 7). It therefore seems likely that the $k a t G$ genes from several mycobacteria are, contrary to those from other bacteria, regulated by FurA in a fashion similar to that ascertained for FurS from Streptomyces reticuli. Thus it would be interesting to test whether clinical mycobacterial strains carry varying mutations in the corresponding fur $A$ genes, leading to differing levels of the catalase-peroxidase KatG and subsequently to different degrees of isoniazid resistance.

Sequencing data indicate that the genome of $\mathrm{Myco}$ bacterium tuberculosis harbours, apart from the Streptomyces reticuli furS homologue, a furB and a furC gene whose deduced gene products are quite closely related to each other, but significantly differ from FurA and Streptomyces reticuli FurS (Fig. 7). The Bacillus 
subtilis genome contains three fur-like genes which encode the repressors Fur (formerly YqkL), PerR (formerly $\mathrm{YgaG}$ ) and $\mathrm{YqfV}$ whose functions have not yet been explored (Bsat et al., 1998). It will also be of interest to investigate whether the genomes of streptomycetes contain two to three fur-like genes, like those of Mycobacterium tuberculosis, Bacillus subtilis, Synechocystis sp. PCC 6803, Deinococcus radiodurans, Thermotoga maritima and Aquifex aeolicus. The analyses (unrooted phylogenetic tree) of all known deduced Fur-like proteins from different bacteria allow us to classify four additional types (Fig. 7) in addition to the 'classic' E. coli Fur type.

Previously a homologue (desR) of $d t x R$ was found in Streptomyces pilosus (Günter-Seeboth \& Schupp, 1995). DtxR had been identified as iron repressor in Corynebacterium diphtheriae (Tao et al., 1994) and it had been assumed that fur genes are missing in streptomycetes. Our data show for the first time that a Streptomyces strain harbours a fur-like gene encoding a functional, regulatory protein. It will be of great interest to define the detailed interaction of the FurS-type protein with divalent first-row transition metal ions and with specific DNA motifs. Our additional hybridization studies suggest that fur-like genes are also present in other Streptomyces species. Our TBLASTN analysis revealed that a genomic fragment, which was sequenced by chance from Streptomyces venezuelae (Fig. 7), harbours a fur-like gene differing from Streptomyces reticuli furS. It is planned to investigate Streptomyces genes regulated by the different types of fur-like genes in more detail.

We are grateful to TIGR, Oklahoma University databases and the Genome Therapeutics Corp., USA, for making their sequence data available prior to publication. We thank $M$. Lemme for her help with the writing of the manuscript and $\mathrm{K}$. Hertel for performing some enzyme assays. The work (including support for the guest scientist I. Borovok, Tel Aviv University, Department of Molecular Microbiology and Biotechnology, Tel Aviv, Israel) was financed by the Deutsche Forschungsgemeinschaft SFB 171/C14.

Allday, M. J. \& Jones, M. D. (1987). Rapid processing of nitrocellulose filter lifts of bacteriophage lambda libraries. Nucleic Acids Res 15, 10592.

Altschul, S. F., Madden, T. L., Schäffer, A. A., Zhang, J., Zhang, Z., Miller, W. \& Lipman, D. J. (1997). Gapped BLAST and PSI-BLAST : a new generation of protein database search programs. Nucleic Acids Res 25, 3389-3402.

Bagg, A. \& Neilands, J. B. (1987). Molecular mechanism of regulation of siderophore mediated iron assimilation. Microbiol Rev 51, 509-518.

Beck, S. \& Pohl, F. M. (1984). DNA sequencing with direct blotting electrophoresis. EMBO J 3, 2905-2909.

Bourke, B., Al Rashid, S. T., Bingham, H. L. \& Chan, V. L. (1996). Characterization of Campylobacter upsaliensis fur and its localization in a highly conserved region of the Campylobacter genome. Gene 183, 219-224.
Bradford, M. M. (1976). A rapid and sensitive method for the quantitation of microgram quantities of protein utilizing the principle of protein-dye binding. Anal Biochem 72, 248-254.

Brunder, W., Schmidt, H. \& Karch, H. (1996). KatP, a novel catalase-peroxidase encoded by the large plasmid of enterohaemorrhagic Escherichia coli O157:H7. Microbiology 142, 3305-3315.

Bsat, N., Herbig, A., Casillas-Martinez, L., Setlow, P. \& Helmann, J. D. (1998). Bacillus subtilis contains multiple Fur homologues: identification of the iron uptake (Fur) and peroxide regulon (PerR) repressors. Mol Microbiol 29, 189-198.

Cendrin, F., Jouve, H. M., Gaillard, J., Thibault, P. \& Zaccai, G. (1994). Purification and properties of a halophilic catalaseperoxidase from Haloarcula marismortui. Biochim Biophys Acta 1209, 1-9.

Conyers, S. M. \& Kidwell, D. A. (1991). Chromogenic substrates for horseradish peroxidase. Anal Biochem 192, 207-211.

Deretic, V., Philipp, W., Dhandayuthapani, S., Mudd, M. H., Curcic, R., Garbe, T., Heym, B., Via, L. E. \& Cole, S. T. (1995). Mycobacterium tuberculosis is a natural mutant with an inactivated oxidative-stress regulatory gene: implications for sensitivity to isoniazid. Mol Microbiol 17, 889-900.

Dittrich, W., Betzler, M. \& Schrempf, H. (1991). An amplifiable and deletable chloramphenicol-resistance determinant of Streptomyces lividans 1326 encodes a putative transmembrane protein. Mol Microbiol 5, 2789-2797.

Dower, W. J., Miller, J. F. \& Ragsdale, C. W. (1988). High efficiency transformation of E. coli by high voltage electroporation. Nucleic Acids Res 16, 6127-6145.

Eiglmeier, K., Fsihi, H., Heym, B. \& Cole, S. T. (1997). On the catalase-peroxidase gene, $k a t G$, of Mycobacterium leprae and the implications for treatment of leprosy with isoniazid. FEMS Microbiol Lett 149, 273-278.

Elstner, E. F. (1982). Oxygen activation and oxygen toxicity. Annu Rev Plant Physiol 33, 73-96.

Finzel, B. C., Poulos, T. L. \& Kraut, J. (1984). Crystal structure of yeast cytochrome $c$ peroxidase refined at $1 \cdot 7$ - $\AA$ resolution. $J$ Biol Chem 259, 13027-13036.

Fukumori, Y., Fujiwara, T., Okada-Takahashi, Y., Mukohata, Y. \& Yamanaka, T. (1985). Purification and properties of a peroxidase from Halobacterium halobium L-33. J Biochem (Tokyo) 98, 1055-1061.

Gold, L., Pribnow, D., Schneider, T., Shninedling, S., Singer, B. S. \& Stormo, G. (1981). Translational initiation in prokaryotes. Annu Rev Microbiol 35, 365-403.

Günter-Seeboth, K. \& Schupp, T. (1995). Cloning and sequence analysis of the Corynebacterium diphtheriae $d t x R$ homologue from Streptomyces lividans and S. pilosus encoding a putative iron repressor protein. Gene 166, 117-119.

Hall, H. K. \& Foster, J.W. (1996). The role of Fur in the acid tolerance response of Salmonella typhimurium is physiologically and genetically separable from its role in iron acquisition. I Bacteriol 178, 5683-5691.

Hantke, K. (1981). Regulation of ferric iron transport in Escherichia coli $\mathrm{K}-12$ : isolation of a constitutive mutant. Mol Gen Genet 182, 288-292.

Hantke, K. (1984). Cloning of the repressor protein gene of iron-regulated systems in Escherichia coli K12. Mol Gen Genet 197, 337-341.

Heidrich, C., Hantke, K., Bierbaum, G. \& Sahl, H. G. (1996). Identification and analysis of a gene encoding a Fur-like protein 
of Staphylococcus epidermidis. FEMS Microbiol Lett 140, 253-259.

Henikoff, S. (1987). Unidirectional digestion with exonuclease III in DNA sequence analysis. Methods Enzymol 155, 156-166.

Higgins, D. G., Bleasby, A. J. \& Fuchs, R. (1992). Clustal v: improved software for multiple sequence alignment. Comput Appl Biosci 8, 189-191.

Hopwood, D. A., Bibb, M. J., Chater, K. F., Kieser, T., Bruton, C. J., Kieser, H. M., Lydiate, D. J., Smith, C. P., Ward, J. M. \& Schrempf, H. (1985). Genetic Manipulation of Streptomyces: a Laboratory Manual. Norwich: John Innes Foundation.

Laemmli, U. K. (1970). Cleavage of structural proteins during the assembly of the head of bacteriophage T4. Nature 227, 680-685.

Loewen, P. C. \& Hengge-Aronis, R. (1994). The role of the sigma factor $(\mathrm{KatF})$ in bacterial global regulation. Annu Rev Microbiol 48, 53-80.

Loprasert, S., Negoro, S. \& Okada, H. (1988). Thermostable peroxidase from Bacillus stearothermophilus. J Gen Microbiol 134, 1971-1976.

Magliozzo, R. S. \& Marcinkeviciene, J. A. (1997). The role of $\mathrm{Mn}(\mathrm{II})$-peroxidase activity of mycobacterial catalase-peroxidase in activation of the antibiotic isoniazid. J Biol Chem 272, $8867-8870$.

Marcinkeviciene, J. A., Magliozzo, R. S. \& Blanchard, J. S. (1995). Purification and characterization of the Mycobacterium smegmatis catalase-peroxidase involved in isoniazid activation. J Biol Chem 270, 22290-22295.

Margoliash, E., Novogrodsky, A. \& Schejter, A. (1960). Irreversible reaction of 3-amino-1:2:4-triazole and related inhibitors with the protein of catalase. Biochem J 74, 339-348.

Nadler, V., Goldberg, I. \& Hochman, A. (1986). Comparative study of bacterial catalases. Biochim Biophys Acta 882, 234-241.

Nagy, J. M., Cass, A. E. \& Brown, K. A. (1995). Progress in the characterization of catalase-peroxidase from Mycobacterium tuberculosis. Biochem Soc Trans 23, 152 S.

Nakata, N., Matsuoka, M., Kashiwabara, Y., Okada, N. \& Sasakawa, C. (1997). Nucleotide sequence of the Mycobacterium leprae kat $G$ region. J Bacteriol 179, 3053-3057.

Nielsen, H., Engelbrecht, J., Brunak, S. \& von Heijne, G. (1997). Identification of prokaryotic and eukaryotic signal peptides and prediction of their cleavage sites. Protein Eng 10, 1-6.

Ochsner, U. A. \& Vasil, M. L. (1996). Gene repression by the ferric uptake regulator in Pseudomonas aeruginosa: Cycle selection of iron-regulated genes. Proc Natl Acad Sci USA 93, 4409-4414.

Pagan-Ramos, E., Song, J., McFalone, M., Mudd, M. H. \& Deretic, V. (1998). Oxidative stress response and characterization of the oxyR-ahpC and furA-katG loci in Mycobacterium marinum. J Bacteriol 180, 4856-4864.

Poulos, T. L., Patterson, W. R. \& Sundaramoorthy, M. (1995). The crystal structure of ascorbate and manganese peroxidases: the role of non-haem metal in the catalytic mechanism. Biochem Soc Trans 23, 228-232.
Pugsley, A. P., Poquet, I. \& Kornacker, M. G. (1991). Two distinct steps in pullulanase secretion by Escherichia coli K12. Mol Microbiol 5, 865-873.

Sambrook, J., Fritsch, E. F. \& Maniatis, T. (1989). Molecular Cloning: a Laboratory Manual, 2nd edn. Cold Spring Harbor, NY: Cold Spring Harbor Laboratory.

Schlochtermeier, A., Niemeyer, F. \& Schrempf, H. (1992a). Biochemical and electron microscopic studies of the Streptomyces reticuli cellulase (Avicelase) in its mycelium-associated and extracellular forms. Appl Environ Microbiol 58, 3240-3248.

Schlochtermeier, A., Walter, S., Schröder, J., Moormann, M. \& Schrempf, H. (1992b). The gene encoding the cellulase (Avicelase) Cel1 from Streptomyces reticuli and analysis of protein domains. Mol Microbiol 6, 3611-3621.

Smulevich, G. (1995). The functional role of the key residues in the active site of peroxidases. Biochem Soc Trans 23, 240-244.

Steinman, H. M., Fareed, F. \& Weinstein, L. (1997). Catalaseperoxidase of Caulobacter crescentus: function and role in stationary-phase survival. J Bacteriol 179, 6831-6836.

Storz, G., Tartaglia, L. A. \& Ames, B. N. (1990). Transcriptional regulator of oxidative stress-inducible genes: direct activation by oxidation. Science 248, 189-194.

Strohl, W. R. (1992). Compilation and analysis of DNA sequences associated with apparent streptomycete promoters. Nucleic Acids Res 20, 961-974.

Tao, X., Schiering, N., Zeug, H. Y., Ringe, D. \& Murphy, J. R. (1994). Iron, DtxR and the regulation of diphtheria toxin expression. Mol Microbiol 14, 191-197.

Vara, J., Lewandowska-Skarbek, M., Wang, Y.-G., Donadio, S. \& Hutchinson, C. R. (1989). Cloning of genes governing the deoxysugar portion of the erythromycin biosynthesis pathway in Saccharopolyspora erythraea (Streptomyces erythreus). I Bacteriol 171, 5872-5881.

Wattiau, P., Woestyn, S. \& Cornelis, G. R. (1996). Customized secretion chaperones in pathogenic bacteria. Mol Microbiol 20, $255-262$.

Wayne, L. G. \& Diaz, G. A. (1986). A double staining method for differentiating between two classes of mycobacterial catalase in polyacrylamide electrophoresis gels. Anal Biochem 157, 89-92.

Welinder, K. G. (1991). Bacterial catalase-peroxidases are gene duplicated members of the plant peroxidase superfamily. Biochim Biophys Acta 1080, 215-220.

West, S., Schröder, J. \& Kunz, W. (1990). A multiple-staining procedure for the detection of different DNA fragments on a single blot. Anal Biochem 190, 254-258.

Zhang, Y., Heym, B., Allen, B., Young, D. \& Cole, S. (1992). The catalase-peroxidase gene and isoniazid resistance of $\mathrm{Myco-}$ bacterium tuberculosis. Nature 358, 591-593.

Zheng, M., Åslund, F. \& Storz, G. (1998). Activation of the OxyR transcription factor by reversible disulphide bond formation. Science 279, 1718-1721.

Received 28 October 1998; revised 23 November 1998; accepted 26 November 1998 